attribute to an "abstinence syndrome." In all of the literature which I have been able to survey, and in personal clinical experience, hallucinations have not been seen as a symptom of psychostimulant withdrawal. The most generally described symptoms are lethargy, depression, and suicide attempt. I would suspect that these symptoms in the case presented represent the psychologic response of a schizophrenic woman to the withdrawal of a drug on which she is psychically dependent, or a psychic response to hospitalization.

Studies have demonstrated the considerable correspondence between very large doses of stimulants over a period of a few days and the development of a paranoid psychosis. The aetiology of a psychosis which has existed for one and a half years at first observation, developing four and a half years after beginning to take excessive quantities of phenmetrazine, is problematic. This is so especially when that dosage, while excessive, is less than that which may be necessary to cause the acute, drug-induced psychosis. If we may roughly equate $25 \mathrm{mg}$. phenmetrazine with $5 \mathrm{mg}$. dexamphetamine, then their patient took the equivalent of $30-40 \mathrm{mg}$. dexamphetamine, daily. This is, in my experience, insufficient to cause an acute drug (or sleep deprivation) psychosis. That chronic, moderately high doses of amphetamines can cause a psychosis independent of personality variables has been suggested, ${ }^{2}$ but not established.-I am, etc.,

Department of Psychiatry,
Beth Israel Medical Center,
New York, N.Y., U.S.A.
References
Griffith, J., Amer. F. Psychiat., 1966, 123, 560
Lemere, F., Amer. f. Psychiat., 1966, 123, 569.

\section{Subdural Haematoma and Effusion in Infancy}

SiR,-Mr. Murray A. Falconer (7 September, p. 614) is indeed correct when he writes that the treatment of subdural haematoma and effusion is sub judice. I believe it will remain so for a long time. He states that the groups which I described, treated by one of the two distinct methods, do not allow "valid statistical comparisons." A re-reading of my paper will show that I did not attempt such comparisons, and was well aware of the pitfalls. I stated that treatment by subdural-pleural shunt "may be superior to craniotomy," and that in the group treated by this method the follow-up period was shorter so that " assessment of impaired intelligence is more difficult and less accurate."

My statement that operation for insertion of a subdural-pleural shunt is simpler and less traumatic than craniotomy is correct. Such features are of importance in the surgery of infancy.

Dr. J. G. Henderson's remarks and questions are very pertinent (14 September, p. 678). My short paper (17 August, p. 400) attempted to deal only with the presentation of the disease and two methods of treatment. It will be recognized by all who have had dealings with these infants that surgical treatment is often only an early incident in the long and difficult management of a family situation. I deliberarely avoided such complex matters in my article, but of course cannot do so in clinical practice.

The psychosocial history about which Dr. Henderson inquires became available in most of $\mathrm{my}$ cases. One important discovery was that no less than $14 \%$ of the infants came from foster homes. In six cases the parents were eventually taken to court accused of injuring the child. In 27 other cases I considered there was reason to suspect that injury had been inflicted intentionally. I much regret the way in which the phrase "battered baby" is so frequently used without definition. I am reasonably sure that some of the babies in my series were injured intentionally by adults on one occasion only and never again. It is unwise and harmful, in my opinion, to call this a "battered baby syndrome "; to do so may induce an attitude in the doctors, nurses, children's officer etc., which impedes our ability to help the parents and the child. I do not think that we have, in Britain, sufficient data to allow profitable discussion in medical journals. Rather should we organize group surveys and studies of the family situations in which these babies are found. Minimum requirements at present include a radiological survey of the whole skeleton and discussions with parents, medical social workers, and local medical officers of health. The possibility that retinal or subhyaloid haemorrhage may be due to injury elsewhere in the body, referred to by Dr. Henderson, is important, but should not be allowed to reduce our vigilance in seeking a cause within the skull.

In the follow-up period only a small proportion of children appear to have been injured again. Such findings will depend upon our care in looking for evidence of injury and (more importantly) upon the measures taken to help the family in avoiding a repetition of the original abuse. To protect the child from further injury (which is sometimes fatal) and yet to avoid removing him unnecessarily from the parents is a difficult task of those who are increasingly concerned with these matters. - I am, etc.,

\section{Department of Neurosurgery London W.C.1.}

\section{KenNeth Till.}

\section{Spout Cervical Oesophagostomy}

SIR,-I was horrified to read the letter by Mr. L. F. Tinckler (17 August, p. 433) on the use of spout cervical oesophagostomy in terminal malignant obstruction of the oesophagus. The author says "one of the distressing terminal features.... is the patient's inability to swallow his saliva."

Surely the author does not seriously suggest that this method is preferable to the many other well-tried and acceptable procedures, ranging from Souttar's tubes, the various makes of plastic tubes, and the Mousseau-Barbin tube, to palliative bypass procedures using tubes of stomach or other bowel, all of which have as their objective the ability of the patient to swallow his own saliva as well as food and drink. As to collecting his discharges and homogenizing them for gastrostomy feeding, the mind boggles. These poor unfortunate people occasionally have a year or two or even longer left of life, but in the majority of cases it is only a matter of months.
In my opinion gastrostomy is never indicated as a palliative treatment (although a case can occasionally be made out for a temporary one in conjunction with other surgery). No doubt other more informed thoracic surgeons than myself will reply to this letter, but I must enter a protest against this operation and suggest that there can be no possible indication for its use.-I am, etc.,

Christopher Cummins.

Toowoomba, Queensland, Australia.

SIR, - The last sentence in your leading article (17 August, p. 392) on orf conveyed the impression that orf in man is a rare disease. Orf, or ovine contagious pustular dermatitis, has been known and recognized as a clinical entity for a long time. It occurs in most of the sheep-rearing countries of the world, and the curious name has regional variants. The Australians call it " scabby mouth," and the hill farmers of Whest Wales call it simply " crach." Its seasonal incidence is fairly constant, and it seems to occur mainly in the spring and early summer. Older lambs and young sheep are most commonly affected. Loss of condition and development of the lambs is said to be due to the orf infection itself, but feeding difficulties often arise from the lesions in the mouths of the lambs and on the teats of the ewes. A mild case with multiple small vesicles and little crust formation can easily be missed in a flock.

In man there are some interesting clinical features. Human orf is recognized more frequently and its significance better understood by shepherds and veterinary surgeons than by doctors. It tends to affect children rather than adults, the farmer's wife perhaps more often than the farmer, and the veterinary student almost as often as the veterinary practitioner. There is seldom any constitutional disturbance with orf in man. This can, however, occur, and in one case biopsy of a lesion appcared to precipitate a viraemia with a generalized maculopapular rash. The bacteriological service in the area has found virological studies of crusts disappointing. Fortunately, clinical diagnoses are usually straightforward, particularly with a history of animal contact. Nevertheless, a physician colleague was once confronted with a case of human orf during a smallpox epidemic. This case was fully investigated virologically before being accepted as orf.

The Figure shows a lesion on the vulva of a child aged 9 which was diagnosed clinically as orf. I was asked to see the patient in May this year. The child's mother had assigned the lesion to the child's habit of riding bareback on her favourite pony. There was orf in the sheep on the farm, and outbreaks had recurred over recent years. The child also fed and played with a pet lamb which had mild orf infection. The vulval lesion presented a typical clinical appearance of human orf. The whole area was reddened and there were nummular lesions on each labia majus, all with raised margins and most with purulent semi-necrotic centres. There were seven in all. There was no bacteriological evidence of secondary infection. The 
inguinal glands on both sides were easily palpable but were neither tender nor enlarged. There was no constitutional disturbance.

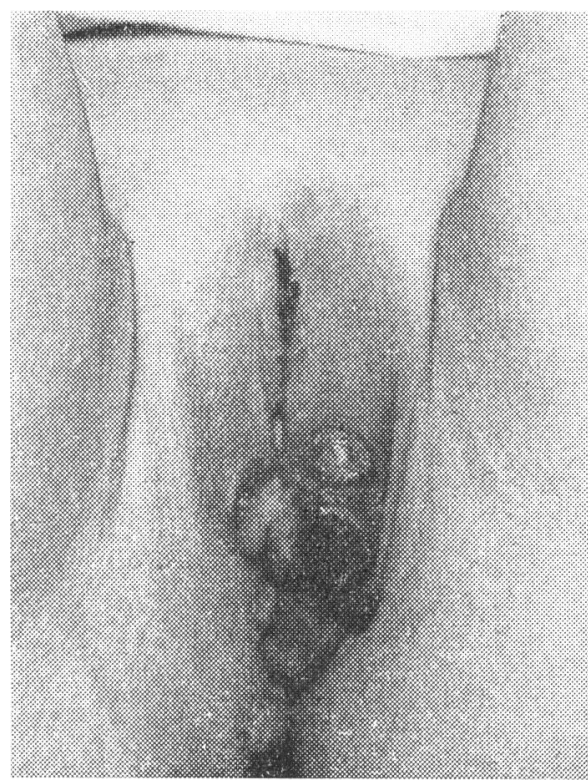

These rather unpleasant-looking lesions slowly resolved without treatment, and had disappeared six weeks from the date of referral.-I am, etc.,

Carmarthen.

\section{J. R. E. JAMES.}

\section{Cerebral Malaria}

SIR,-I am interested in the recent correspondence (27 July, p. 250 ; and 14 September, p. 680) regarding cerebral malaria.

I spent some nine years in East Pakistan where $P$. falciparum is endemic and was able to treat a fair number of cases during that time. The most striking thing about a case of the disease is the rapidity of its deterioration once coma has set in. Many patients may come to a remote country hospital or dispensary from some distance in extremis. Need of treatment is paramount. Delays while lumbar punctures and drips are prepared may be fatal.

My earlier experience of using intravenous quinine as the sole parenteral medication was frightening. Two cases on successive days died. However, Manson-Bahr in his invaluable Tropical Diseases advocates the use of intramuscular adrenaline. From then onwards the standard treatment in our small hospital was as follows: Intravenous quinine dihydrochloride $10 \mathrm{gr}$. in $10 \mathrm{ml}$. distilled water given slowly over 10 minutes. Immediatel; thereafter $1 \mathrm{ml}$. $1: 10,000$ adrenaline intramuscularly over five minutes. We treated probably about a dozen cases without a single fatality by this method. It would appear that just as the steroids have supplanted adrenaline in bronchial asthma so they are doing in cerebral malaria.

The rationale of the adrenaline treatment would appear to be threefold. (1) It causes the spleen to contract, expelling parasites into the blood stream and facilitating the action of the quinine; (2) it causes peripheral vasoconstriction, and this aids the resuscitation of the patient who is always collapsed, with poor peripheral circulation; and (3) it has an effect on the cerebral oedema mainly because of its vasoconstrictive action. Needless to say, other supportive measures such as ice bags and drips are necessary, but my experience would suggest that the consistent use of quinine and adrenaline saves time. The other measures will aid recovery.

Dr. T. Harding need not fear his budget will be stretched. Adrenaline is an essential item of equipment in every general practitioner's bag, let alone in a small hospital, and it is relatively cheap, compared with dexamethasone.-I am, etc.,

\section{T. Patrick.}

Bilton,

\section{Chemotherapy of Malaria}

SIR,-I have to-day received the B.M.F. (13 July, p. 71) and read with interest the leading article on chemotherapy of malaria. It is certainly true that in Tanzania at least there have been no substantiated cases of malaria resistant to 4-aminoquinolines. There have, however, for a long time been many areas where there has been resistance to both pyrimethamine and proguanil, so much so that for many years we have completely given up using these drugs in any form of malaria, either for treatment of an acute attack or for chemoprophylaxis.

I think, therefore, it might have been best if this was explained in the second paragraph of your leading article, from which one would understand that pyrimethamine and proguanil should still be used as chemoprophylaxis. I would also have preferred the use of the word chemoprophylaxis or causal prophylaxis rather than chemosuppression. I feel sure that if proguanil and pyrimethamine continue to be used as prophylactics where resistance to these drugs has developed this will not only cause an increase in the incidence of resistant strains of $P$. falciparum but also considerable morbidity and loss of work. The literature is also clear that there have been a not-negligible number of fatal $P$. falciparum malaria in those who have used the above drugs as chemoprophylactics.

So far as our experience goes, we have continued to use the 4-aminoquinolines in both prophylaxis and treatment of malaria and have run into no trouble as a result of this. The position as regards malaria in Tanzania has been fully documented. ${ }^{2}-\mathrm{I}$ am, etc.,

\section{Mvumi Hospital, Tanzania. \\ Clyde, D. F., Malaria in Tanzania, 1967.
London. \\ Joseph TAYLOR.}

\section{Stress and Bronchitis}

Sir,-Dr. A. C. Stenhouse (3 August, p. 287), reporting on acute exacerbations of chronic bronchitis, reports on 22 episodes of "non-specific bronchitis" confined to a few of the patients under study. We would like to point to an emerging factor of significance in the study of upper respiratory infection and other illness that may account for the selection of this and other subgroups, given equivalent virological data and knowledge of their endocrine state. Though the age of the particular patients in question is not given, the data of Dr. Stenhouse's prior study (19 August 1967, p. 461) would place them above the age range of our study; however, this would appear to make the concept no less valid, given the state of knowledge about geriatrics.

Acute upper respiratory infection has come under closer scrutiny with current virological, epidemiological, and endocrinological study. Observation has shown that the average number of colds per person each year is remarkably constant, but that considerable variation is noted in the number of colds experienced annually by both individuals and families. ${ }^{1}$ It has been postulated that genetic factors might govern innate susceptibility or resistance. ${ }^{1}$ Host susceptibility, of whatever origin, appears to be a factor in the relative frequency of subclinical and clinical infections. ${ }^{2}$ A mechanism of susceptibility involving endocrine function on a cellular level has been hypothesized. ${ }^{3}$ The recent endocrine balance study of Mason et al..$^{5}$ showed a number of pre-illness hormonal changes, including an abrupt spike of 17-OHCS, epinephrine, and norepinephrine, and a gradual drop in butanol-extractable iodine. Sick subjects with upper respiratory infections differed from less sick by having a greater number of extreme values. The psychoanalytic literature has presented dynamic formulations that may bear on susceptibility, especially related to conflict situations, ${ }^{6}$ and M. Jackel before the fall 1967 meeting of the American Psychoanalytic Association implicated depression, as had others previously.

The menstrual cycle has well-documented phasic hormonal changes. ${ }^{7}$ As part of an ongoing study at this laboratory it was found that levels of characterological anxiety, or anxiety trait, were associated with physical-emotionalsocial impairment during the premenstruum. Inquiry as to the incidence of upper respiratory infection in the studied population of 161 female student nurses showed a significantly greater number of acute upper respiratory infections in the "high" trait anxious group than in the "low" anxiety group. This relationship to trait anxiety, as measured by the Taylor Manifest Anxiety Scale, was significant at the 0.01 level. Measures of situational anxiety, or anxiety state (the Scheier Cattell Anxiety Battery), and of depression (the " $D$ " scale of the Minnesota Multiphasic Personality Inventory) were not significant in differentiating the populations with and without colds. There was not a significant correlation between colds and impairment independent of anxiety.

A synthesis of the data from various fields indicates that there should be a discriminating factor in the incidence of upper respiratory infection. The study of premenstrual tension has provided a naturally occurring "stress" that has documented hormonal changes that fit with previously delineated mechanisms of infection. Since all women undergo hormonal changes during the menstrual cycle, the factor of significance, however subtle it may be, is the influence of psychic response to such "stress." Response characteristics may accentuate or modify the pattern of hormonal change in the menstruum, thereby enhancing susceptibility. This accentuation or modification in the individual may be based on a characteristic response to acute stress of defined type. The observation of a significant relationship between trait anxiety and the incidence of upper respiratory infection represents a start toward the better definition of individual difference as a significant factor in the onset of illness. This finding can aid in understanding such findings as those of Dr. Stenhouse, where appar- 